\title{
EVALUACIÓN SEMICUANTITATIVA DE GLUCÓGENO MEGACARIOCÍTICO PARA EL DIAGNÓSTICO DE PÚRPURA TROMBOCITOPÉNICA IDIOPÁTICA
}

\author{
Semiquantitative assessment of megakaryocytes glycogen for diagnosis idiopathic thrombo- \\ cytopenic purpura
}

Juan M. Parreño T ${ }^{1}$, Luz F. Oyola $\mathrm{H}^{2}$ y Anselma Pardo $\mathrm{S}^{3}$.

${ }^{1}$ Unidad de Post-Grado - Fac. de Farmacia y Bioquímica, UNMSM, ${ }^{2}$ Dpto. Acad. de Bioquímica, Facultad de Farmacia y Bioquímica, UNMSM ${ }^{3}$ Centro de Análisis Bioquímicos y Clínicos, UNW.

\section{RESUMEN}

El objetivo del presente estudio fue determinar semicuantitativamente glucógeno megacariocítico y los parámetros afines como ayuda al diagnóstico y tratamiento de la púrpura trombocitopénica idiopática (PTI). Las pruebas se aplicaron a 100 pacientes con diagnóstico de PTI provenientes de diversos hospitales y clínicas y en 100 sujetos aparentemente sanos, quienes dieron su consentimiento informado de acuerdo a la Declaración de Helsinki. Se investigó la presencia de glucógeno en los megacariocitos del frotis de médula ósea usando el método del ácido peryódico de Schiff y exámenes complementarios de la hemostasia: recuento de plaquetas por el método de Rees-Ecker, volumen plaquetario medio (VPM) utilizando el analizador automático Sysmex XE-210o, tiempo de protrombina, tiempo parcial de tromboplastina (TPT), tiempo de sangría, fibrinógeno, hemoglobina y recuento de glóbulos rojos por los métodos usuales. Se concluye que los pacientes con PTI presentaban disminución de glucógeno megacariocítico (100\%); alteraciones en algunos de los parámetros de la coagulación como: aumento del tiempo de sangría (98\%), disminución del número de plaquetas (100\%); manteniéndose los tiempos de protrombina, TPT y fibrinógeno, y aumento en el VPM (88\%). Además, se encontró una correlación positiva y significativa entre los valores disminuidos de plaquetas y el VPM.

Palabras clave: Púrpura trombocitopénica idiopática, glucógeno megacariocítico, megacariocitos.

\section{SUMMARY}

The aim of this study was to determine semiquantitatively megakaryocyte glycogen and related parameters as an aid to diagnosis and treatment of idiopathic thrombocytopenic purpura (ITP). The tests were applied to 100 patients diagnosed with ITP from various hospitals and clinics, and 100 apparently healthy subjects who was gave informed consent according to the Declaration of Helsinki. Investigating the presence of glycogen in megakaryocytes of bone marrow smears using the method of peryodic acid-Schiff and complementary tests of hemostasis: platelets by the method of Rees-Ecker, mean platelet volume (MPV) using the automatic analyzer Sysmex XE-210o, prothrombin time, partial thromboplastin time (PTT), bleeding time, fibrinogen, hemoglobin and red blood cell count by the usual methods. We conclude that patients with ITP megakaryocyte glycogen had decreased (100\%), alterations in some coagulation parameters such as increased bleeding time (98\%), decreased number of platelets (100\%) remaining prothrombin time, PTT and fibrinogen, and increased MPV (88\%). In addition, we found a significant positive correlation between decreased levels of platelets and the MPV.

Keywords: Idiopathic thrombocytopenic purpura, glycogen megakaryocyte, megakaryocyte.

\section{INTRODUCCIÓN}

a púrpura trombocitopénica idiopática (PTI) es una enfermedad hemorrágica autoinmune $^{(1)}$ que se caracteriza por el desarrollo de autoanticuerpos antiplaquetarios (IgG) dirigidos contra antígenos de algunos de los complejos mayores de glicoproteínas plaquetarias (GpIIb/IIIa, GpIb/IX/V o GpIa/IIa, GpIV), las cuales son destruidas por fagocitosis en el bazo, debido a que éste no reco- noce a las propias plaquetas y en consecuencia las destruye, y con menor frecuencia en el hígado. En el adulto, la enfermedad es gradual y fluctuante sin ningún síntoma precedente, con un curso crónico mayor a 6 meses y puede ser aguda en un pequeño porcentaje. La gran mayoría de los pacientes requieren tratamiento farmacológico a base de glucocorticoides, quimioterapia inmunosupresora y en casos extremos esplenectomía ${ }^{(2)}$. Se considera una enfermedad de mecanismo inmunológico idiopático ${ }^{(3)}$. 
Las plaquetas ayudan a que la sangre se coagule, aglutinándose para taponar pequeños agujeros en los vasos sanguíneos dañados, son metabólicamente activas, contienen peptidasa, nucleotidasa, fosfatasa alcalina y fosfatasa ácida en cantidades considerables; pirofosfatasa y actividad de deshidrogenasa. Las granulaciones de los trombocitos contienen polisacáridos como el glucógeno, y el hialómero segrega retractócima ${ }^{(4,5)}$.

No se han encontrado formas inmaduras de las plaquetas, sino más bien una disminución de éstas; no hay falta de producción, pero si una velocidad exagerada en su autodestrucción, lo que concuerda con el déficit de glucógeno que se presenta a nivel del megacariocito ${ }^{(6)}$.

El Volumen Plaquetario Medio (VPM) es una magnitud calculada por el analizador, que expresa el tamaño promedio de las plaquetas. Las plaquetas "nuevas" son más grandes, por lo que se presenta aumento del VPM cuando se produce un mayor número de plaquetas, siendo este un medio de información acerca de la producción de plaquetas por la médula ósea ${ }^{(7,8)}$.

La investigación comprende los estudios citoquímico, hematológico y bioquímico en aspirado de médula ósea y sangre venosa, tanto en los pacientes con PTI como en los sujetos control, con el objetivo de hallar la causa de esta patología y poder así, no sólo establecer los protocolos para el diagnóstico y tratamiento de esta enfermedad, sino confirmar la deficiencia de glucógeno intracitoplasmático en los megacariocitos de los pacientes con PTI.

\section{MATERIALES Y MÉTODOS}

\section{Muestras biológicas}

\section{Médula ósea}

Las muestras fueron obtenidas por el especialista mediante aspiración de la médula ósea, de preferencia de la cresta iliaca posterior. A partir del aspirado, se realizaron extensiones en láminas portaobjetos para la evaluación semicuantitativa de glucógeno en los megacariocitos por el método del ácido peryódico de Schiff (PAS) ${ }^{(9,10)}$. Los frotis fueron secados durante 30 minutos al aire y luego fijados antes de la reacción citoquímica.

\section{Muestras de sangre}

- Sangre venosa: Extraída de la vena cubital media de la flexura del codo y recibida en tubo de ensayo sin anticoagulante y frasco conteniendo el anticoagulante de Wintrobe.
- Sangre capilar: Extraída del pulpejo del dedo y del lóbulo de la oreja.

\section{Población de estudio}

- Grupo de estudio: Conformado por 100 pacientes adultos con diagnóstico clínico de PTI.

- Grupo control: Conformado por 100 sujetos adultos aparentemente sanos, a quienes se les descartó clínicamente PTI.

\section{Estudio citoquímico en médula ósea}

Investigación de glucógeno. Se utilizó el método de PAS ${ }^{(11,12)}$.

Fundamento: El ácido peryódico es un oxidante que rompe los enlaces $\mathrm{C}-\mathrm{C}$ vecinos de polisacáridos (glucógeno), si los dos átomos de carbono llevan grupos hidroxilos se oxidan a aldehídos, los que se combinan con el reactivo de Schiff para dar un producto de color rojo púrpura. Presenta reacción positiva de color rojo púrpura para el glucógeno de los megacariocitos y también es positiva para las plaquetas ${ }^{(13,14)}$.

\section{ESTUDIOS HEMATOLÓGICOS}

Recuento de plaquetas ${ }^{(11,15)}$. Método directo de ReesEcker. V.N.: 150 ooo-400 ooo plaquetas por mm.

Volumen plaquetario medio (VPM) ${ }^{(15,16)}$. Método automatizado. V.N.: 7,4-10,4 fL.

Tiempo de sangría o de hemorragia ${ }^{(3,15)}$. Método de Duke. V.N.: 1-3 minutos.

Tiempo de protrombina ${ }^{(15,16)}$. Método Ortho-Brain. V.N.: 11-13 segundos.

Tiempo parcial de tromboplastina (TPT) (3,16). Macrométodo de una sola fase. V.N.: 30-45 segundos.

\section{ESTUDIO BIOQUÍMICO}

Dosaje de fibrinógeno ${ }^{(1,15)}$. Método de Gornall Bardawill y David. V.N.: 200-400 mg/dL.

\section{RESULTADOS}

Tabla 1. Glucógeno en megacariocitos de aspirado de médula ósea.

\begin{tabular}{ccc}
\hline \multirow{2}{*}{ Reacción de PAS } & \multicolumn{2}{c}{ Grupo } \\
\cline { 2 - 3 } & Problema & Control \\
\hline Glucógeno disminuído o negativo & $100 \%$ & $0 \%$ \\
Glucógeno positivo & $0 \%$ & $100 \%$ \\
Total & $100 \%$ & $100 \%$ \\
\hline
\end{tabular}

Chi cuadrado: $201,45 \quad p=0,000<0,05$ 
Tabla 2. Investigación semicuantitativa de glucógeno.

\begin{tabular}{|c|c|c|c|c|c|}
\hline \multicolumn{6}{|c|}{ Grupo } \\
\hline \multicolumn{3}{|c|}{ Problema } & \multicolumn{3}{|c|}{ Control } \\
\hline $\begin{array}{c}\text { Reacción } \\
\text { de PAS }\end{array}$ & $\begin{array}{l}\text { Número } \\
\text { de casos }\end{array}$ & Porcentaje & $\begin{array}{c}\text { Reacción } \\
\text { de PAS }\end{array}$ & $\begin{array}{l}\text { Número } \\
\text { de casos }\end{array}$ & Porcentaje \\
\hline+ & 58 & $58 \%$ & ++ & 4 & $4 \%$ \\
\hline \multirow[t]{3}{*}{-} & 42 & $42 \%$ & +++ & 66 & $66 \%$ \\
\hline & 100 & $100 \%$ & ++++ & 30 & $30 \%$ \\
\hline & & & & 100 & $100 \%$ \\
\hline
\end{tabular}

Tabla 4. Correlación del recuento de plaquetas y volumen paquetario medio en el grupo problema.

\begin{tabular}{llc}
\hline & Correlación Pearson & $\begin{array}{c}\text { Volumen } \\
\text { plaquetario medio }\end{array}$ \\
\hline \multirow{2}{*}{ Recuento de Plaquetas } & $\mathrm{p}$ & 0,437 \\
& Número de casos & 0,000 \\
& 100 \\
\hline
\end{tabular}

Correlación positiva y significativa $(0,437 \mathrm{P}<0,05)$

Tabla 5. Comparación de promedio en parámetros hematológicos y bioquímicos en los grupos proble ontroles $(\mathrm{n}=100)$.

\begin{tabular}{clcccc}
\hline & & Media $^{+}$ & $\begin{array}{c}\text { Desviación } \\
\text { estándar }\end{array}$ & Mínimo & Máximo \\
\hline T. de sangría* & Problema & 17,30 & 4,69 & 10,00 & 30,00 \\
& Control & 5,70 & 1,79 & 3,00 & 9,00 \\
$\begin{array}{c}\text { T. de } \\
\text { protrombina* }\end{array}$ & Problema & 12,20 & 1,01 & 10,00 & 14,00 \\
$\begin{array}{c}\text { T. parcial de } \\
\text { tromboplastina* }\end{array}$ & Problema & 40,88 & 6,97 & 4,00 & 50,00 \\
& Control & 35,50 & 4,05 & 30,00 & 45,00 \\
$\begin{array}{c}\text { Recuento de } \\
\text { plaquetas* }\end{array}$ & Problema & 37800,00 & 9507,04 & 20000,00 & 57000,00 \\
& Control & 317160,00 & 95431,05 & 180000,00 & 450000,00 \\
$\begin{array}{c}\text { Fibrinógeno* } \\
\text { Volumen }\end{array}$ & Problema & 291,60 & 44,92 & 190,00 & 490,00 \\
plaquetario & Problema & 12,51 & 1,83 & 7,90 & 16,40 \\
\hline medio* & Control & 9,42 & 0,69 & 7,80 & 10,30 \\
\hline p<0,05 diferencias significativas & & $+\mathrm{t}$ Student & & \\
\hline
\end{tabular}

\section{DISCUSIÓN}

En 1953, Storti y col. ${ }^{(17)}$ demostraron la presencia de granulaciones de glucógeno en la periferie de los megacariocitos en muestras de médula ósea. Daniel ${ }^{(18)}$ en estudios realizados en extensiones coloreadas de médula ósea de pacientes con PTI, observó la presen-
Tabla 3. Porcentaje de parámetros hematológicos y bioquímicos en los grupos problema y control.

\begin{tabular}{|c|c|c|c|}
\hline & & \multicolumn{2}{|c|}{ Grupo } \\
\hline & & Problema & Control \\
\hline \multirow{3}{*}{$\begin{array}{l}\text { Recuento de } \\
\text { plaquetas } \\
\text { Chi cuadrado } 177,35 \\
\mathrm{p}=0,000<0,05\end{array}$} & Disminuido & $100 \%$ & $6 \%$ \\
\hline & Normal & $0 \%$ & $92 \%$ \\
\hline & Aumentado & $0 \%$ & $2 \%$ \\
\hline \multirow{3}{*}{$\begin{array}{l}\text { Tiempo de } \\
\text { sangría } \\
\text { Chi cuadrado 96,o7 } \\
\mathrm{p}=0,000<0,05\end{array}$} & Disminuido & $0 \%$ & $0 \%$ \\
\hline & Normal & $2 \%$ & $100 \%$ \\
\hline & Aumentado & $98 \%$ & $0 \%$ \\
\hline \multirow{3}{*}{$\begin{array}{l}\text { Tiempo de } \\
\text { protrombina } \\
\text { Chi cuadrado } 1,84 \\
p=0,39>0,05\end{array}$} & Disminuido & $6 \%$ & $2 \%$ \\
\hline & Normal & $86 \%$ & $94 \%$ \\
\hline & Aumentado & $8 \%$ & $4 \%$ \\
\hline \multirow{3}{*}{$\begin{array}{l}\text { Tiempo parcial de } \\
\text { tromboplastina } \\
\text { Chi cuadrado } 8,33 \\
\mathrm{p}=0,01<0,05\end{array}$} & Disminuido & $2 \%$ & $0 \%$ \\
\hline & Normal & $92 \%$ & $100 \%$ \\
\hline & Aumentado & $6 \%$ & $0 \%$ \\
\hline \multirow{3}{*}{$\begin{array}{l}\text { Fibrinógeno } \\
\text { Chi cuadrado } 4,59 \\
\mathrm{p}=0,10>0,05\end{array}$} & Disminuido & $4 \%$ & $0 \%$ \\
\hline & Normal & $90 \%$ & $96 \%$ \\
\hline & Aumentado & $6 \%$ & $4 \%$ \\
\hline \multirow{3}{*}{$\begin{array}{l}\text { Volumen } \\
\text { plaquetario medio } \\
\text { Chi cuadrado 157,14 } \\
\mathrm{p}=0 \text {, ooo }<0,05\end{array}$} & Disminuido & $0 \%$ & $0 \%$ \\
\hline & Normal & $12 \%$ & $100 \%$ \\
\hline & Aumentado & $88 \%$ & $0 \%$ \\
\hline
\end{tabular}

cia de glucógeno en forma de pequeñas manchas intracitoplasmáticas en las plaquetas, que fueron confirmadas por Jamra y Lorenzi ${ }^{(19)}$ en un estudio adicional de glucógeno megacariocítico. Desde entonces no se han reportado estudios en relación a este compuesto intracitoplasmático a nivel de los megacariocitos y las plaquetas.

En la presente investigación se ha demostrado que, en los megacariocitos de la médula ósea las concentraciones de glucógeno están disminuidas (+) en un $58 \%$ o ausentes (-) en un $42 \%$ (tablas 1 y 2), lo cual está estrechamente relacionado con una disminución considerable de plaquetas (tabla 3); esto nos sugiere relacionar la deficiencia de glucógeno como una de las posibles causas de esta enfermedad, constituyéndose como una prueba de gran valor, no sólo para el diagnóstico sino también para el tratamiento de la PTI por existir una relación directa entre la concentración del glucógeno y la actividad del megacariocito. 
El aporte de la citoquímica ha permitido el hallazgo de una serie de compuestos bioquímicos en los elementos formes de la sangre, así como explicar la etiología de la PTI mediante el uso de la prueba de PAS (3). En personas sanas existe una relación entre la concentración del glucógeno y la actividad del megacariocito, y las plaquetas presentan glucógeno positivo ${ }^{(11)}$.

Con respecto a las otras pruebas hematológicas y bioquímicas se encontró que, el tiempo de sangría estaba prolongado en el $98 \%$ de los casos (tabla 3 ) debido a su estrecha relación con las plaquetas que ayudan al taponamiento de la salida de sangre. El tiempo de protrombina y el tiempo parcial de tromboplastina (tabla 3) están dentro de los niveles normales, ya que en la formación de fibrina, los factores de coagulación no interfieren en la actividad de las plaquetas ${ }^{(1)}$.

Las concentraciones de fibrinógeno (tabla 3) son normales en el 90\% de los casos de PTI, y esto es explicable debido a que el fibrinógeno plasmático no está influenciado por los factores que promueven la destrucción de las plaquetas.

Es importante destacar que, el volumen plaquetario (tablas 3 y 4) se encontró incrementado en el $88 \%$ de los casos con PTI; esto se explica debido a una respuesta de los mecanismos homeostáticos del organismo a fin de compensar la disminución acelerada de plaquetas, permitiendo la salida de aquellas en fase de maduración y anormalmente grandes.

Al comparar los parámetros de los casos con PTI versus controles, se encontró diferencias significativas $(\mathrm{p}<0.05)$ en diferentes variables. Los tiempos de: sangría, protrombina y parcial de tromboplastina son ligeramente mayores; el número de plaquetas y valores de fibrinógeno son ligeramente menores y el volumen plaquetario medio es mayor (tabla 5).

Por los resultados obtenidos en el presente estudio pensamos que muy pronto dejaría de utilizarse el adjetivo "idiopático" de la PTI, toda vez que una de sus causas es la deficiencia de glucógeno en los megacariocitos.

\section{REFERENCIA BIBLIOGRÁFICAS}

1. Henry J. El laboratorio en el diagnóstico clínico. 1oa ed. Edit. Marbán. Madrid, 2007.

2. Kumar S, Dieh M, Gertz A, Tefferi A. Splenectomy for immune thrombocytopenic purpura: Long-term results and treatment of postsplenectomy relapses. Hematol Intern Med. 2002; 1:312-7.

3. Deska K, Pagana T. Guía de pruebas diagnósticas y de laboratorio. 8a ed. Edit. Elsevier. Barcelona, 2008.
4. Velez H, Rojas W, Borrero J, Restrepo J. Fundamento de Medicina. Hematología. 5a ed. Corporación para investigaciones biológicas. Medellín, 2001.

5. Sans S. Hematología Clínica. 5a ed. Edit. Elsevier. Madrid, 2006.

6. Galano GP. Idiopathic thrombocytopenic purpura is a relatively common autoimmune hemorrhagic disease. A retrospective study was conducted among 141 patients that received attention from 1983 to 2003. Aimed at. 2004; 241:110-7.

7. Sánchez M, Anitua E, Azofra J, Andía I, Padilla S, Mujika I. Comparison of surgically repaired Achilles tendon tears using platelet-rich fibrin matrices. The American Journal of Sports Medicine. 2007; 35 (2): 245-51.

8. Diccionario Médico Biológico. za ed. Edit. Panamericana. México DF, 1998.

9. Lilleyman JS. Intracarnial haemorragie in idiopathic thrombocytopenic purpura. Arch Dis Child, 1994; 71: 251-253.

10. Liendo F. Púrpura trombocitopénica autoinmune. 2a ed. Edit. Mediterráneo. Santiago de Chile, 1997.

11. Vives JLl, Aguilar JLl. Manual de técnicas de laboratorio en hematología. za ed. Edit. Elsevier-Masson. Madrid, 2006.

12. Agurto T, Ramos J. Técnicas de coloración de células y tejidos. 5a ed. Edit. Imprenta Unión. Lima, 2007.

13. Reid MH. Bone marrox examination before steroides in thrombocytopenic purpura and artritis. Acta Pediatr 1992; 81: 1052-1053.

14. Cripe TP. Idiopathic thrombocytopenic purpura. Reasons to resolve the case. J. Pediatr Hematol Oncol. 1999; 21 (6): 465-466.

15. Talaska F. Manual de pruebas diagnósticas. 7a ed. Edit. McGraw-Hill Interamericana. México DF, 2002.

16. Gonzáles De Buitrago JM. Técnicas y métodos de laboratorio clínico. $2^{\mathrm{da}}$ ed. Edit. Masson. Barcelona, 2005.

17. Storti E, Perugino S, Soldati M. Cytochemical investigations of normal megakaryocytes and platelets. Biotechnic and histochemistry. 1990; 65(5): 211 - 230.

18. Daniel HW. Studies of megakaryocyte glycogen. Semiquantitative Method of Measurement: Effect of Phlebotomies in Young Adults. American Society of Hematology, 1959, Vol. 14, No. 1, pp. 6o-73.

19. Fauci AS, Braunwald E, et al. Harrisson. Principios de Medicina Interna. 17a ed. Edit. Mc Graw Hill. Madrid, 2001.

Manuscrito recibido el: 12/12/2009

Aceptado para su publicación el: 16/02/2010

Correspondencia:

Nombre: Juan M. Parreño Tipian

Dirección: Jr. Tacna 233 Chorrillos

e-mail: jupartip21@hotmail.com 